\title{
Sensing-Throughput Tradeoff for Cognitive Radio Systems with Unknown Received Power
}

\author{
Ankit Kaushik ${ }^{1}$, Shree Krishna Sharma ${ }^{2}$, Symeon Chatzinotas ${ }^{2}$, Björn \\ Ottersten $^{2}$, and Friedrich Jondral ${ }^{1}$ \\ 1 Communications Engineering Lab, Karlsruhe Institute of Technology (KIT), \\ Germany, \\ \{ankit.kaushik, friedrich.jondral\}@kit.edu \\ ${ }^{2}$ SnT - securityandtrust.lu, University of Luxembourg, Luxembourg, \\ \{shree.sharma, symeon. chatzinotas, bjorn.ottersten\}@uni.lu
}

\begin{abstract}
Understanding the performance of the cognitive radio systems is of great interest. Different paradigms have been extensively analyzed in the literature to perform secondary access to the licensed spectrum. Of these, Interweave System (IS) has been widely investigated for performance analysis. According to IS, sensing is employed at the Secondary Transmitter (ST) that protects the Primary Receiver (PR) from the interference induced. Thus, in order to control the interference at the $\mathrm{PR}$, it is required to sustain a certain level of probability of detection. In this regard, ST requires the knowledge of the received power. However, in practice, this knowledge is not available at the ST. Thereby performing analysis considering the prior knowledge of the received power is too idealistic, thus, do not depict the actual performance of the IS. Motivated by this fact, an estimation model that includes received power estimation is proposed. Considering a sensing-throughput tradeoff, we apply this model to characterize the performance of the IS. Most importantly, the proposed model captures the estimation error to determine the distortion in the system performance. Based on analysis, it is illustrated that the ideal model overestimates the performance of the IS. Finally, it is shown that with an appropriate choice of the estimation time, the severity in distortion can be effectively regulated.
\end{abstract}

\section{Introduction}

For future wireless technologies, cognitive radio communication is emerging as a possible solution to the problem of spectrum scarcity. The available cognitive radio paradigms in the literature can be categorized into interweave, underlay and overlay [1]. In Interweave Systems (IS), the Secondary Users (SUs) utilize the licensed spectrum opportunistically by exploiting spectral holes in different domains such as time, frequency, space and polarization, whereas in Underlay Systems (US), SUs are allowed to use the primary spectrum as long as they respect the interference constraints of the Primary Receivers (PRs). On the other hand, Overlay Systems (OS) allow the spectral coexistence of two or more wireless networks by employing advanced transmission and coding strategies. 
Due to its ease in deployment, IS is mostly preferred for performing analysis among these paradigms. In this context, this paper focuses on the performance analysis of the ISs considering a hardware deployment where sensing is employed at the Secondary Transmitter (ST).

\subsection{Motivation}

Sensing is an integral part of the IS. At the ST, sensing is necessary for detecting the presence and absence of a primary signal, thereby protecting the PRs against harmful interference. Sensing at the ST is accomplished by listening to the transmission from the PT. For detecting a primary signal, several techniques such as Energy Detection (ED), matched filtering, cyclostationary and feature-based detection exist $[2,3]$. Because of its versatility towards unknown primary signals, $\mathrm{ED}$ has been extensively investigated in the literature $[4,5,6,7,8]$. According to $\mathrm{ED}$, the decision is accomplished by comparing the power received at the ST to a threshold. In reality, the ST encounters a variation in the received power due to the thermal noise at the receiver and fading in the channel. This leads to sensing errors described as misdetection or false alarm. The characterization of sensing errors as probability of detection and probability of false alarm has been studied in [9]. These sensing errors limit the performance of the IS.

In particular, probability of detection is critical for the primary system because it precludes the PR from the interference induced by the ST. On the other side, probability of false alarm accounts for the throughput attained by the secondary system at the Secondary Receiver (SR). In this regard, ST has to sustain a desired probability of detection and optimize its throughput. This phenomenon is characterized as a sensing-throughput tradeoff by Liang et al. [10]. According to it, the ST is able to determine a suitable sensing time that achieves an optimum throughput for a given received power. Several contributions have considered the performance of IS based on sensing errors $[10,11,12]$. However, the analysis described in the literature is too idealistic and not feasible for deployment, as it considers the perfect knowledge of the received power at the ST.

With the presence of channel and noise in the system, the received power is never known accurately, thus, needs to be estimated at the ST. Considering a hardware deployment, it is important to determine the performance of the IS based on received power estimation. A similar analysis is performed in [13], where the authors employ the received power estimation to control transmit power at the ST deployed as an underlay system. However, in this paper, we intend to capture the effect of estimation on the performance of an IS. Now, to realize received power estimation at the $\mathrm{ST}$, it is necessary to allocate a certain time interval for the estimation within the frame duration. With the introduction of this estimation time, the system performance differs from its ideal behaviour. Additionally, the employed estimation process itself induces a certain level of error in the system. Hence, in order to understand the performance of the IS, it is necessary to consider the aforementioned aspects. 


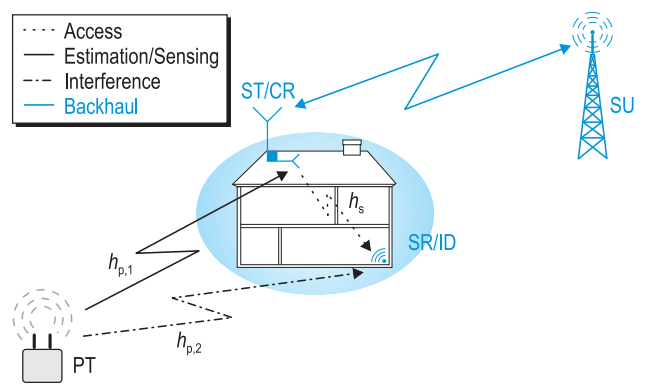

(a)

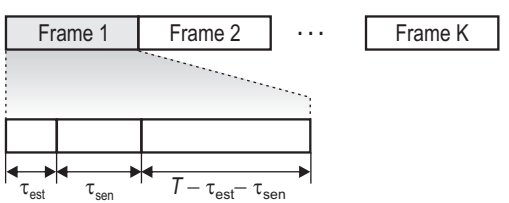

(b)

Fig. 1. (a) A scenario demonstrating the interweave paradigm. (b) Frame structure of interweave system with received power estimation.

\subsection{Contributions}

To realize the received power estimation, we consider a new frame structure. According to it, in a single frame, the ST performs (i) received power estimation, (ii) sensing, and (iii) data transmission. To perform analysis based on this new frame structure, we propose a novel estimation model. Most importantly, we evaluate the system performance with the inclusion of the estimation time and the errors occurred due to estimation. Based on the analytical expressions, we analyze the sensing-throughput tradeoff for the proposed estimation model. Finally, we determine the confidence intervals for the estimated received power. Particularly, based on these intervals, we capture the distortion in the performance based on the upper and lower bounds. This distortion, however, depends on the choice of design parameters depicted as probability of confidence and estimation duration.

\subsection{Organization}

The rest of the paper is organized as follows: Section 2 describes the system model that includes the interweave scenario and the signal model. Section 3 investigates the sensing-throughput tradeoff for the estimation model and derives upper and lower bounds for the performance parameters. Section 4 analyzes the numerical results based on the obtained expressions. Finally, Section 5 concludes the paper.

\section{System Model}

\subsection{Interweave scenario}

Cognitive Relay (CR) [14] characterizes a small cell deployment that fulfills the spectral requirements for indoor devices. Fig. 1a illustrates a snapshot of a CR scenario to depict the interaction between the $\mathrm{CR}$ with PT and indoor device, where CR and indoor device represent ST and SR, respectively. In [14], the challenges involved while deploying the $\mathrm{CR}$ as an IS were presented. For 
simplification, a constant false alarm rate was considered in the system model. Now, we extend the analysis to employ a constant detection rate.

The medium access for the IS is slotted, where the time axis is segmented into frames of length $T$. The frame structure is analog to the one illustrated in [10]. However, unlike [10], the proposed frame structure uses $\tau_{\text {est }}$ to estimate and $\tau_{\text {sen }}$ to sense the received power, where $\tau_{\text {est }}, \tau_{\text {sen }}$ correspond to time intervals and $\tau_{\text {est }}+\tau_{\text {sen }}<T$, cf. Fig. 1b. To incorporate the effect of fading in the model, we assume that the channel remains constant for $T$. Hence, characterized by the fading process, each frame witnesses a different received power. Therefore, to sustain a desired probability of detection, it is important to perform estimation $\tau_{\text {est }}$ followed by sensing $\tau_{\text {sen }}$ for each frame. The remaining time $T-\left(\tau_{\text {est }}+\tau_{\text {sen }}\right)$ is utilized for data transmission.

\subsection{Signal model}

In the estimation and sensing phase, the received signal at the ST is sampled with a sampling frequency of $f_{\mathrm{s}}$ for given hypotheses, that depicts the presence $\left(\mathcal{H}_{1}\right)$ and absence $\left(\mathcal{H}_{0}\right)$ of the primary signal, is given by

$$
y_{\mathrm{rcvd}}[n]= \begin{cases}\sqrt{h_{\mathrm{p}, 1}} \cdot x_{\mathrm{p}}[n]+w[n] & : \mathcal{H}_{1} \\ w[n] & : \mathcal{H}_{0}\end{cases}
$$

where $x_{\mathrm{p}}[n]$ corresponds to a discrete sample at the PT, $h_{\mathrm{p}, 1}$ represents the power gain for the channel and $w[n]$ is circularly symmetric complex Additive White Gaussian Noise (AWGN) at the ST. $x_{\mathrm{p}}[n]$ is an i.i.d. (independent and identically distributed) random process. As the channel $h_{\mathrm{p}, 1}$ is independent to $x_{\mathrm{p}}$ and $w[n]$ is an i.i.d. Gaussian random process with zero mean and variance $\mathbb{E}\left[|w[n]|^{2}\right]=\sigma^{2}$, therefore, $y_{\mathrm{rcvd}}[n]$ corresponds to an i.i.d. random process. The true received power is defined as

$$
\bar{P}_{\mathrm{rcvd}}=\mathbb{E}\left[\left|\sqrt{h_{\mathrm{p}, 1}} \cdot x_{\mathrm{p}}[n]\right|^{2}\right] .
$$

Based on (2), the received SNR at the ST is $\gamma_{\mathrm{rcvd}}=\frac{\bar{P}_{\mathrm{rcvd}}}{\sigma^{2}}-1$.

Now, the data transmission at the ST is conditioned over the probability of detection $\left(\mathrm{P}_{\mathrm{d}}\right)$. In this context, the received signal at the $\mathrm{SR}$ is given by

$$
y_{\mathrm{s}}[n]= \begin{cases}\sqrt{h_{\mathrm{s}}} \cdot x_{\mathrm{s}}[n]+\sqrt{h_{\mathrm{p}, 2}} \cdot x_{\mathrm{p}}[n]+w[n] & : 1-\mathrm{P}_{\mathrm{d}} \\ \sqrt{h_{\mathrm{s}}} \cdot x_{\mathrm{s}}[n]+w[n] & : \mathrm{P}_{\mathrm{d}},\end{cases}
$$

where $x_{\mathrm{s}}[n]$ is an i.i.d. random process and corresponds to discrete signal transmitted by the ST. Further, $h_{\mathrm{s}}$ and $h_{\mathrm{p}, 2}$ represent the power gains for channel, cf. Fig. 1a. The received SNRs over the links ST-SR and PT-ST are $\gamma_{\mathrm{s}}=\frac{\mathbb{E}\left[\left|\sqrt{h_{\mathrm{s}}} \cdot x_{\mathrm{s}}[n]\right|^{2}\right]}{\sigma^{2}}$ and $\gamma_{\mathrm{p}}=\frac{\mathbb{E}\left[\left|\sqrt{h_{\mathrm{p}, 2}} \cdot x_{\mathrm{p}}[n]\right|^{2}\right]}{\sigma^{2}}$. $[4]$

In the estimation phase, the estimated power received at the ST is given as 


$$
P_{\text {rcvd }}=\frac{1}{\tau_{\text {est }} f_{\mathrm{s}}} \sum_{n}^{\tau_{\text {est }} f_{\mathrm{s}}}\left|y_{\mathrm{rcvd}}[n]\right|^{2} \text {. }
$$

$P_{\text {rcvd }}$ determined in (4) using $\tau_{\text {est }} f_{\mathrm{s}}$ samples follows a non central chi-squared distribution [15]. Considering large number of samples, thereby following similar approach as in [9], we apply the central limit theorem to approximate the distribution for $P_{\text {rcvd }}$ as Gaussian distribution

$$
P_{\text {rcvd }} \sim \mathcal{N}\left(\bar{P}_{\text {rcvd }}, \frac{2}{\tau_{\text {est }} f_{\mathrm{s}}} \bar{P}_{\text {rcvd }}^{2}\right)
$$

Following the estimation of the received power, the ST performs sensing for a duration of $\tau_{\text {sen }}$, cf. Fig. 1b. The test statistics $T(\mathbf{y})$ at the ST is evaluated as

$$
T(\mathbf{y})=\frac{1}{\tau_{\text {sen }} f_{\mathrm{s}}} \sum_{n}^{\tau_{\text {sen }} f_{\mathrm{s}}}\left|y_{\text {rcvd }}[n]\right|^{2} \underset{\mathcal{H}_{0}}{\stackrel{\mathcal{H}_{1}}{\gtrless}} \epsilon,
$$

where $\epsilon$ is the threshold and $\mathbf{y}$ is a vector with $\tau_{\text {sen }} f_{\mathrm{s}}$ samples. The probability of detection $\mathrm{P}_{\mathrm{d}}$ and the probability of false alarm $\mathrm{P}_{\mathrm{fa}}$ corresponding to (6) are determined as [9]

$$
\begin{gathered}
\mathrm{P}_{\mathrm{d}}\left(\epsilon, \tau_{\mathrm{sen}}, \bar{P}_{\mathrm{rcvd}}\right)=\mathcal{Q}\left(\frac{\epsilon-\bar{P}_{\mathrm{rcvd}}}{\sqrt{\frac{2}{\tau_{\mathrm{sen} f_{\mathrm{s}}}}} \bar{P}_{\mathrm{rcvd}}}\right), \\
\mathrm{P}_{\mathrm{fa}}\left(\epsilon, \tau_{\mathrm{sen}}\right)=\mathcal{Q}\left(\frac{\epsilon-\sigma^{2}}{\sqrt{\frac{2}{\tau_{\mathrm{sen} f_{\mathrm{s}}}} \sigma^{2}}}\right),
\end{gathered}
$$

where $\mathcal{Q}(\cdot)$ represents the Q-function [16]. Subsequently, by sustaining the $\mathrm{P}_{\mathrm{d}}$ above a certain desired level $\overline{\mathrm{P}}_{\mathrm{d}}$

$$
\mathrm{P}_{\mathrm{d}}\left(\epsilon, \tau_{\text {sen }}, \bar{P}_{\text {rcvd }}\right) \geq \overline{\mathrm{P}}_{\mathrm{d}},
$$

the ST precludes the interference to the primary system. Consequently, an optimum performance is achieved when the ST operates at the desired level, i.e., $\mathrm{P}_{\mathrm{d}}=\overline{\mathrm{P}}_{\mathrm{d}}$. Hence, using (7) and (9), the threshold is evaluated as

$$
\epsilon\left(\overline{\mathrm{P}}_{\mathrm{d}}, \tau_{\text {sen }}, \bar{P}_{\mathrm{rcvd}}\right)=\left(\mathcal{Q}^{-1}\left(\overline{\mathrm{P}}_{\mathrm{d}}\right) \sqrt{\frac{2}{\tau_{\text {sen }} f_{\mathrm{s}}}}+1\right) \bar{P}_{\text {rcvd }}
$$

\subsection{Assumptions}

As a preliminary step, for the proposed model, we consider only the estimation of $\bar{P}_{\text {rcvd }}$ at the ST. Hence, in this paper, it is assumed that the ST acquires the perfect knowledge about $\gamma_{\mathrm{p}}$ and $\gamma_{\mathrm{s}}$ from the SR over a feedback channel. The 
inclusion of the imperfect knowledge of $\gamma_{\mathrm{p}}$ and $\gamma_{\mathrm{s}}$ in the proposed model poses an interesting research direction. Moreover, we consider that all transmitted signals are subjected to distance dependent path loss and the small scale fading gains. The coherence time for the channel gain is greater than the frame duration. However, we may still encounter scenarios where the coherence time exceeds the frame duration, in such cases our characterization depicts a lower performance bound. With no loss of generality, we consider that the channel gain $\left(h_{\mathrm{p}, 1}, h_{\mathrm{p}, 2}\right.$ and $h_{\mathrm{s}}$ ) includes the distance dependent path loss and the small scale gain. Finally, we target short term performance, according to which the performance parameters are optimized for each frame.

\section{Sensing-Throughput analysis}

\subsection{Ideal Model (IM)}

According to Liang et al. [10], the secondary throughput subject to a desired probability of detection $\overline{\mathrm{P}}_{\mathrm{d}}$ is given by

$$
\begin{aligned}
& \begin{aligned}
& \tilde{R}_{\mathrm{s}}\left(\tilde{\tau}_{\mathrm{sen}}\right)=\max _{\tau_{\mathrm{sen}}} R_{\mathrm{s}}\left(\tau_{\mathrm{sen}}\right)=\frac{T-\tau_{\mathrm{sen}}}{T}\left[C_{0}\left(1-\mathrm{P}_{\mathrm{fa}}\left(\epsilon, \tau_{\mathrm{sen}}\right)\right) \mathrm{P}\left(\mathcal{H}_{0}\right)\right. \\
&\left.+C_{1}\left(1-\mathrm{P}_{\mathrm{d}}\left(\epsilon, \tau_{\mathrm{sen}}, \bar{P}_{\mathrm{rcvd}}\right)\right) \mathrm{P}\left(\mathcal{H}_{1}\right)\right], \\
& \text { s.t. } \mathrm{P}_{\mathrm{d}}\left(\epsilon, \tau_{\mathrm{sen}}, \bar{P}_{\mathrm{rcvd}}\right) \geq \overline{\mathrm{P}}_{\mathrm{d}},
\end{aligned} \\
& \text { where } C_{0}=\log _{2}\left(1+\gamma_{\mathrm{s}}\right) \text { and } C_{1}=\log _{2}\left(1+\frac{\gamma_{\mathrm{s}}}{\gamma_{\mathrm{p}}+1}\right) .
\end{aligned}
$$

$\mathrm{P}\left(\mathcal{H}_{0}\right)$ and $\mathrm{P}\left(\mathcal{H}_{1}\right)$ are the probabilities of occurrence for the respective hypothesis. Based on (11), the ST is able to determine the suitable sensing time $\tau_{\text {sen }}=\tilde{\tau}_{\text {sen }}$ such that an optimum throughput $\tilde{R}_{\mathrm{s}}\left(\tilde{\tau}_{\text {sen }}\right)$ is achieved. According to (11), the performance parameters for the IM are defined as $\tilde{R}_{\mathrm{s}}, \mathrm{P}_{\mathrm{d}}$ and $\mathrm{P}_{\mathrm{fa}}$.

\subsection{Estimation Model (EM)}

The system described in [10] is good for performing analysis, however, to determine $\tilde{\tau}_{\text {sen }}$ at the ST requires the knowledge of the received power $\bar{P}_{\text {rcvd }}$. Considering a hardware deployment, this information is not available at the ST. Unless estimated, it is not possible to determine $\tilde{\tau}_{\text {sen }}$. According to the EM, the ST estimates the $\bar{P}_{\text {rcvd }}$ for a duration of $\tau_{\text {est }}$ as $P_{\text {rcvd }}$ and based on its value, the ST determines $\tilde{\tau}_{\text {sen }}$ for the given frame. The samples needed for estimation can be utilized for sensing as well. However, for analytical tractability, in the proposed model the estimation and sensing are considered to be disjoint in time. Now, with the introduction of $\tau_{\text {est }}$, the actual performance of the IS deviates from its ideal performance. Moreover, the estimation itself causes distortion in the actual performance of the IS. As a part of the proposed model, these aspects are dealt in the following subsections. 


\subsection{Actual performance}

With the introduction of the EM, we first characterize the performance of the IS. To realize this, it is considered that the ST perfectly estimates the $P_{\text {rcvd }}$, that is $P_{\text {rcvd }}=\bar{P}_{\text {rcvd }}$. In accordance with the proposed model, the sensing-throughput tradeoff with perfect estimation is determined as

$$
\begin{gathered}
\tilde{R}_{\mathrm{s}}^{\mathrm{P}}\left(\tilde{\tau}_{\text {sen }}^{\mathrm{P}}\right)=\max _{\tau_{\text {sen }}} R_{\mathrm{s}}^{\mathrm{P}}\left(\tau_{\text {sen }}\right)=\frac{T-\left(\tau_{\text {est }}+\tau_{\text {sen }}\right)}{T}\left[C_{0}\left(1-\mathrm{P}_{\text {fa }}\left(\epsilon, \tau_{\text {sen }}\right)\right)\right. \\
\left.\mathrm{P}\left(\mathcal{H}_{0}\right)+C_{1}\left(1-\mathrm{P}_{\mathrm{d}}\left(\epsilon, \tau_{\text {sen }}, \bar{P}_{\text {rcvd }}\right)\right) \mathrm{P}\left(\mathcal{H}_{1}\right)\right], \\
\text { s.t. } \mathrm{P}_{\mathrm{d}}\left(\epsilon, \tau_{\text {sen }}, \bar{P}_{\text {rcvd }}\right) \geq \overline{\mathrm{P}}_{\mathrm{d}} .
\end{gathered}
$$

According to the (12), for a given $\overline{\mathrm{P}}_{\mathrm{d}}$ and estimation of $\bar{P}_{\text {rcvd }}$ in the interval $\tau_{\text {est }}, \mathrm{ST}$ is able to determine the threshold as $\epsilon\left(\overline{\mathrm{P}}_{\mathrm{d}}, \tau_{\mathrm{sen}}, \bar{P}_{\mathrm{rcvd}}\right)$. Finally, based on the new sensing-throughput tradeoff (12), ST evaluates the suitable sensing time as $\tau_{\text {sen }}=\tilde{\tau}_{\text {sen }}^{\mathrm{P}}$ that achieves the optimum throughput $\tilde{R}_{\mathrm{s}}^{\mathrm{P}}$. However, $\tilde{\tau}_{\text {sen }} \neq \tilde{\tau}_{\text {sen }}^{\mathrm{P}}$ due to the inclusion of the estimation time in the considered sensing-throughput tradeoff. According to the EM, the performance parameters that characterize the actual performance of the IS are $\tilde{R}_{\mathrm{s}}^{\mathrm{P}}, \mathrm{P}_{\mathrm{d}}^{\mathrm{P}}$ and $\mathrm{P}_{\text {fa }}^{\mathrm{P}}$. Now, with the perfect estimation of $P_{\mathrm{rcvd}}$, the constraint in (12) is sustained, hence, $\mathrm{P}_{\mathrm{d}}=\overline{\mathrm{P}}_{\mathrm{d}}$. However, with $\tilde{\tau}_{\text {sen }} \neq \tilde{\tau}_{\text {sen }}^{\mathrm{P}}, \mathrm{P}_{\text {fa }}^{\mathrm{P}}$ and $\tilde{R}_{\mathrm{s}}^{\mathrm{P}}$ witness a deviation from their ideal behaviour.

\subsection{Distortion}

Previously, we determined the effect of estimation time on the performance. In this section, we extend the analysis by considering the influence of estimation error on the system performance. In this context, based on (5), we characterize a confidence interval $\left[P_{\text {rcvd }}^{\mathrm{L}}, P_{\mathrm{rcvd}}^{\mathrm{U}}\right]$ for a certain choice of probability of confidence $\mathrm{P}_{\mathrm{c}}$ and $\tau_{\text {est }}$ as

$$
P_{\mathrm{rcvd}}=\left\{\begin{array}{l}
P_{\mathrm{rcvd}}^{\mathrm{L}}=\left(\mathcal{Q}^{-1}\left(\frac{\mathrm{P}_{\mathrm{c}}+1}{2}\right) \sqrt{\frac{2}{\tau_{\text {est }} f_{\mathrm{s}}}}+1\right) \bar{P}_{\mathrm{rcvd}} \\
P_{\mathrm{rcvd}}^{\mathrm{U}}=\left(\mathcal{Q}^{-1}\left(1-\frac{\mathrm{P}_{\mathrm{c}+1}}{2}\right) \sqrt{\frac{2}{\tau_{\text {est }} f_{\mathrm{s}}}}+1\right) \bar{P}_{\mathrm{rcvd}}
\end{array}\right.
$$

where $\mathcal{Q}^{-1}(\cdot)$ is the inverse-Q function [16]. Hence, we utilize this confidence interval to depict the maximum estimation error in the estimated received power, thereby characterizing maximum distortion in the performance parameters $\mathrm{P}_{\mathrm{d}}^{\mathrm{P}}$, $\mathrm{P}_{\text {fa }}^{\mathrm{P}}$ and $\tilde{R}_{\mathrm{s}}^{\mathrm{P}}$. For $\mathrm{P}_{\mathrm{c}}=0.95$, the $P_{\mathrm{rcvd}}^{\mathrm{L}}$ and $P_{\text {rcvd }}^{\mathrm{U}}$ are equivalent to the lower and upper bounds of the $\bar{P}_{\text {rcvd }}$.

This confidence interval further depicts the distortion in the system parameters $\epsilon$ and $\tilde{\tau}_{\text {sen }}^{\mathrm{P}}$. Hence, as an intermediate step, we first characterize the distortion in terms of these system parameters. Subject to the received powers as $P_{\text {rcvd }}^{\mathrm{L}}$ and $P_{\text {rcvd }}^{\mathrm{U}}$, the expressions for the threshold are evaluated as

$$
\epsilon^{\mathrm{L}}=\epsilon\left(\overline{\mathrm{P}}_{\mathrm{d}}, \tau_{\text {sen }}, P_{\mathrm{rcvd}}^{\mathrm{L}}\right) \text { and } \epsilon^{\mathrm{U}}=\epsilon\left(\overline{\mathrm{P}}_{\mathrm{d}}, \tau_{\mathrm{sen}}, P_{\mathrm{rcvd}}^{\mathrm{U}}\right) .
$$


Clearly, due to difference in received power estimated at the ST, the expressions in (14) differ from the one illustrated in (10). By inserting the thresholds $\epsilon^{\mathrm{L}}$ and $\epsilon^{\mathrm{U}}$ in (12), the suitable sensing times computed at the ST are represented as

$$
\tilde{\tau}_{\text {sen }}^{\mathrm{L}} \text { and } \tilde{\tau}_{\text {sen }}^{\mathrm{U}} \text {. }
$$

As a result, (14) and (15) clearly illustrates the distortion in the system parameters. Now, as a final step, we characterize the distortion in the performance parameters in terms of the distorted system parameters and the true received power. Consequently, we represent the distortion in $\tilde{R}_{\mathrm{s}}^{\mathrm{P}}, \mathrm{P}_{\mathrm{d}}^{\mathrm{P}}$ and $\mathrm{P}_{\text {fa }}^{\mathrm{P}}$ as a function of $\left(\tilde{\tau}_{\text {sen }}^{\mathrm{L}}, \epsilon^{\mathrm{L}}\right)$ and $\left(\tilde{\tau}_{\text {sen }}^{\mathrm{U}}, \epsilon^{\mathrm{U}}\right)$ subject to true received power, i.e., $\bar{P}_{\text {rcvd }}$.

Following the above discussion, the distortion in the $\mathrm{P}_{\mathrm{d}}^{\mathrm{P}}$, in terms of upper and lower bounds, due to the inclusion of estimation error in the received power is determined as

$$
\mathrm{P}_{\mathrm{d}}^{\mathrm{P}}=\left\{\begin{array}{l}
\mathrm{P}_{\mathrm{d}}^{\mathrm{L}}=\mathrm{P}_{\mathrm{d}}\left(\epsilon^{\mathrm{L}}, \tilde{\tau}_{\mathrm{sen}}^{\mathrm{L}}, \bar{P}_{\mathrm{rcvd}}\right) \\
\mathrm{P}_{\mathrm{d}}^{\mathrm{U}}=\mathrm{P}_{\mathrm{d}}\left(\epsilon^{\mathrm{U}}, \tilde{\tau}_{\mathrm{sen}}^{\mathrm{U}}, \bar{P}_{\mathrm{rcvd}}\right) .
\end{array}\right.
$$

It is evident that the distortion in the $\mathrm{P}_{\mathrm{d}}^{\mathrm{P}}$ results in the violation of the regulatory constraint, c.f. (9). If this constraint is not sustained, it may result in harmful interference at the PR. Hence, using (16), we are able to characterize the situations where the IS may degrade the performance of the primary system.

On similar basis, the distortion in $\mathrm{P}_{\mathrm{fa}}^{\mathrm{P}}$ in terms of upper and lower bounds is depicted as

$$
\mathrm{P}_{\mathrm{fa}}^{\mathrm{P}}=\left\{\begin{array}{l}
\mathrm{P}_{\mathrm{fa}}^{\mathrm{L}}=\mathrm{P}_{\mathrm{fa}}\left(\epsilon^{\mathrm{L}}, \tilde{\tau}_{\mathrm{sen}}^{\mathrm{L}}\right) \\
\mathrm{P}_{\mathrm{fa}}^{\mathrm{U}}=\mathrm{P}_{\mathrm{fa}}\left(\epsilon^{\mathrm{U}}, \tilde{\tau}_{\mathrm{sen}}^{\mathrm{U}}\right) .
\end{array}\right.
$$

Finally, including the distortion in the probabilities $\mathrm{P}_{\mathrm{d}}^{\mathrm{P}}$ and $\mathrm{P}_{\mathrm{fa}}^{\mathrm{P}}$ from (16) and (17) and the system parameters $\left(\epsilon, \tilde{\tau}_{\text {sen }}^{\mathrm{P}}\right)$, the distortion in the optimum throughput $\tilde{R}_{\mathrm{s}}^{\mathrm{P}}$ in terms of upper and lower bounds is determined as

$$
\tilde{R}_{\mathrm{s}}^{\mathrm{P}}=\left\{\begin{array}{l}
\tilde{R}_{\mathrm{s}}^{\mathrm{L}}=\frac{T-\left(\tau_{\mathrm{est}}+\tilde{\tau}_{\mathrm{sen}}^{\mathrm{L}}\right)}{T}\left[C_{0}\left(1-\mathrm{P}_{\mathrm{fa}}\left(\epsilon^{\mathrm{L}}, \tau_{\mathrm{sen}}^{\mathrm{L}}\right)\right) \mathrm{P}\left(\mathcal{H}_{0}\right)\right. \\
+C_{1}\left(1-\mathrm{P}_{\mathrm{d}}\left(\epsilon^{\mathrm{L}}, \tilde{\tau}_{\mathrm{sen}}^{\mathrm{L}}, \bar{P}_{\mathrm{rcvd}}\right) \mathrm{P}\left(\mathcal{H}_{1}\right)\right] \\
\tilde{R}_{\mathrm{s}}^{\mathrm{U}}=\frac{T-\left(\tau_{\text {est }}+\tilde{\tau}_{\mathrm{sen}}^{\mathrm{U}}\right)}{T}\left[C_{0}\left(1-\mathrm{P}_{\mathrm{fa}}\left(\epsilon^{\mathrm{U}}, \tau_{\mathrm{sen}}^{\mathrm{U}}\right)\right) \mathrm{P}\left(\mathcal{H}_{0}\right)\right. \\
+C_{1}\left(1-\mathrm{P}_{\mathrm{d}}\left(\epsilon^{\mathrm{U}}, \tilde{\tau}_{\mathrm{sen}}^{\mathrm{U}}, \bar{P}_{\mathrm{rcvd}}\right) \mathrm{P}\left(\mathcal{H}_{1}\right)\right] .
\end{array}\right.
$$

Based on the expressions (16), (17) and (18) characterized by the EM, it is possible to depict the maximum distortion for the IS from its actual performance. Moreover, the severity in distortion can be controlled through $\mathrm{P}_{\mathrm{c}}$ and $\tau_{\text {est }}$. In particular, it is important to select $\tau_{\text {est }}$ appropriately such that the distortion in the performance doesn't exceed beyond a certain level. This aspect is investigated more deeply in the next section. 


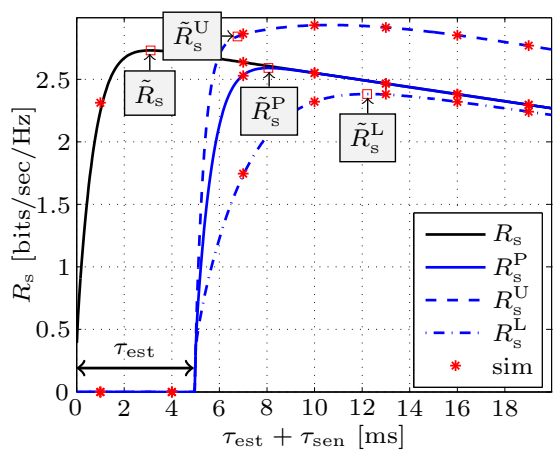

Fig. 2. Sensing-throughput tradeoff for the ideal and estimation models with $\gamma_{\mathrm{rcvd}}=$ $-10 \mathrm{~dB}$ and $\tau_{\text {est }}=5 \mathrm{~ms}$.

\section{Numerical Analysis}

In this section, the performance of the IS for the EM is analyzed. In this regard, we perform simulations to: (i) validate the expressions obtained in Section 3, (ii) provide a mathematical justification to the Gaussian approximation considered in Section 2. Although, the expressions derived using our sensing-throughput analysis are general and applicable to all cognitive radio systems, however, the parameters are selected in such a way that they closely relate to the deployment scenario described in Fig. 1a. Unless stated explicitly, the following choice of the parameters is considered for the analysis, $f_{\mathrm{s}}=1 \mathrm{MHz}, h_{\mathrm{p}, 1}=h_{\mathrm{p}, 2}=$ $-100 \mathrm{~dB}, h_{\mathrm{s}}=-80 \mathrm{~dB}, T=100 \mathrm{~ms}, \mathrm{P}_{\mathrm{c}}=0.95, \overline{\mathrm{P}}_{\mathrm{d}}=0.9, \sigma^{2}=-100 \mathrm{dBm}$, $\gamma_{\mathrm{rcvd}}=-10 \mathrm{~dB}, \gamma_{\mathrm{p}}=-10 \mathrm{~dB}, \gamma_{\mathrm{s}}=10 \mathrm{~dB}$ and $\mathrm{P}\left(\mathcal{H}_{1}\right)=1-\mathrm{P}\left(\mathcal{H}_{0}\right)=0.2$, $\tau_{\text {est }}=5 \mathrm{~ms}$.

Firstly, the analysis in terms of sensing-throughput tradeoff based on (11) and (12) for the IM and the EM is performed. The curves $R_{\mathrm{S}}$ and $R_{\mathrm{s}}^{\mathrm{P}}$ in Fig. 2 depict the throughput based on the IM and the EM (actual performance) at the ST. Due to the inclusion of received power estimation in the frame structure, the ST produces no throughput at the SR for the interval $\tau_{\text {est }}$. The sensing times $\tilde{\tau}_{\text {sen }}=3.11 \mathrm{~ms}$ and $\tilde{\tau}_{\text {sen }}^{\mathrm{P}}=3.06 \mathrm{~ms}$ are evaluated, which yield the optimum throughputs as $\tilde{R}_{\mathrm{s}}=2.73 \mathrm{bits} / \mathrm{sec} / \mathrm{Hz}$ and $\tilde{R}_{\mathrm{s}}^{\mathrm{P}}=2.59 \mathrm{bits} / \mathrm{sec} / \mathrm{Hz}$, cf. Fig. 2 . This variation is due the inclusion of $\tau_{\text {est }}$ in the sensing-throughput analysis. Hence, for the given choice of $\tau_{\text {est }}$ at ST, the ideal model overestimates the optimum throughput by $\approx 5 \%$.

Next, sensing-throughput analysis is performed, considering that the $\bar{P}_{\text {rcvd }}$ is estimated as $P_{\mathrm{rcvd}}^{\mathrm{L}}$ or $P_{\mathrm{rcvd}}^{\mathrm{U}}$ at ST. $\gamma_{\mathrm{rcvd}}=-10 \mathrm{dBm}$ corresponds to $\bar{P}_{\mathrm{rcvd}}=$ $1.10 \cdot 10^{-10} \mathrm{~mW}$. With $\mathrm{P}_{\mathrm{c}}=0.95$ and $\tau_{\text {est }}=5 \mathrm{~ms}$, the confidence intervals are determined as $P_{\mathrm{rcvd}}^{\mathrm{L}}=1.05 \cdot 10^{-10} \mathrm{~mW}$ and $P_{\mathrm{rcvd}}^{\mathrm{U}}=1.14 \cdot 10^{-10} \mathrm{~mW}$, cf. (13). Fig. 2 demonstrates the throughput corresponding to distorted system parameter $\epsilon^{\mathrm{L}}$ and $\epsilon^{\mathrm{U}}$, cf. (14). The suitable sensing times are evaluated as $\tilde{\tau}_{\text {sen }}^{\mathrm{L}}=7.21 \mathrm{~ms}$ and $\tilde{\tau}_{\mathrm{sen}}^{\mathrm{U}}=1.76 \mathrm{~ms}$. Finally, the distortion in the $\tilde{R}_{\mathrm{s}}^{\mathrm{P}}$ corresponding to the distortion in the system parameters is demonstrated. According to (18), the lower bound and upper bound on the optimum throughput $\tilde{R}_{\mathrm{s}}^{\mathrm{P}}$ are determined as 


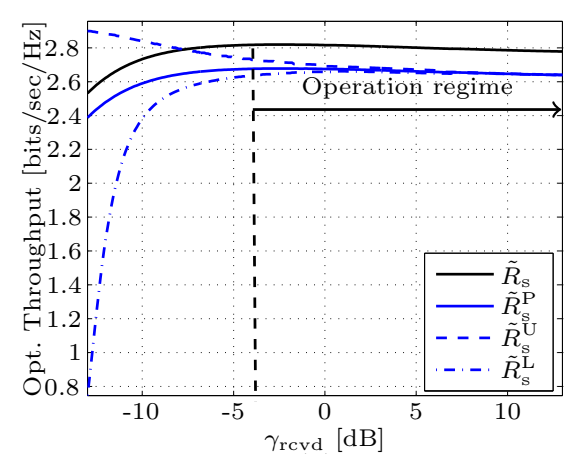

(a)

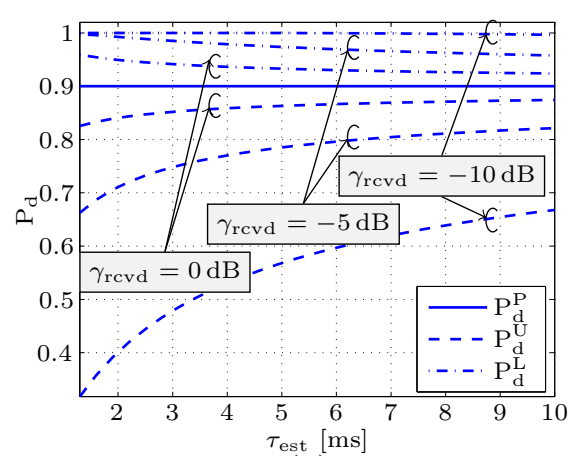

(b)

Fig. 3. (a) Distortion in optimum throughput versus the $\gamma_{\text {rcvd }}$ with $P_{c}=0.95$ and $\tau_{\text {est }}=5 \mathrm{~ms}$. (b) Distortion in probability of detection versus the estimation time for different $\gamma_{\mathrm{rcvd}}=\{-10,-5,0\} \mathrm{dB}$.

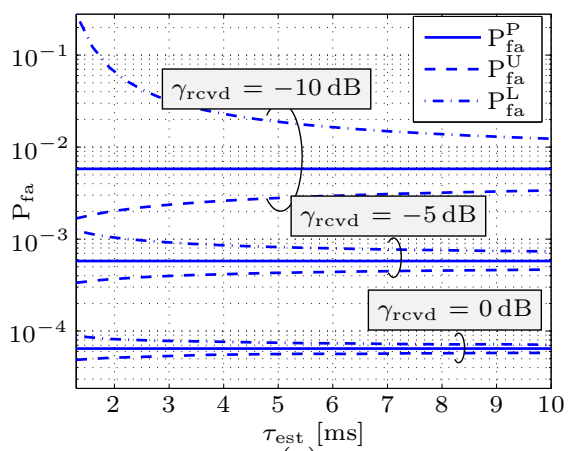

(a)

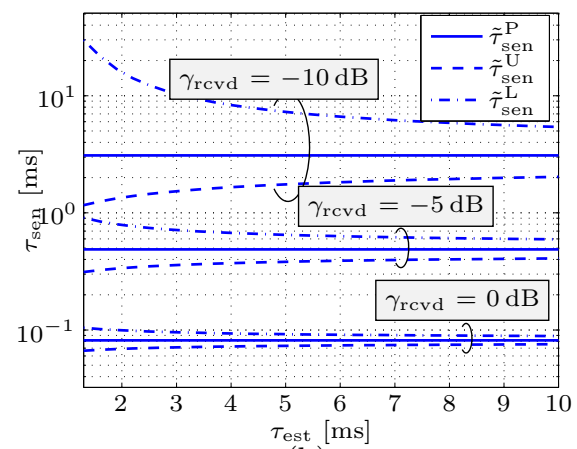

(b)

Fig. 4. (a) Distortion in probability of false versus the estimation time for different $\gamma_{\text {rcvd }} \in\{-10,-5,0\} \mathrm{dB}$. (b) Distortion in optimum sensing time versus the estimation time for different $\gamma_{\mathrm{rcvd}} \in\{-10,-5,0\} \mathrm{dB}$.

$\tilde{R}_{\mathrm{s}}^{\mathrm{L}}=2.38 \mathrm{bits} / \mathrm{sec} / \mathrm{Hz}$ and $\tilde{R}_{\mathrm{s}}^{\mathrm{U}}=2.84 \mathrm{bits} / \mathrm{sec} / \mathrm{Hz}$. It corresponds to $8.10 \%$ underestimation and $9.65 \%$ overestimation of the actual performance $\left(\tilde{R}_{\mathrm{s}}^{\mathrm{P}}\right)$. Therefore, based on the analytical expressions determined under EM, it is possible to determine the severity of distortion in the system performance.

Hereafter, for the analysis, we consider the theoretical expressions and choose to operate at suitable sensing time. Next, we determine the variation of the optimum throughput against the $\gamma_{\mathrm{rcvd}} \in[-13,10] \mathrm{dB}$ at ST with $\tau_{\text {est }}=5 \mathrm{~ms}$. It is evident from Fig. $3 \mathrm{a}$ that the distortion in the $\tilde{R}_{\mathrm{s}}^{\mathrm{P}}$ decreases with increase in $\gamma_{\mathrm{rcvd}}$. For $\gamma_{\mathrm{rcvd}}>-5 \mathrm{~dB}$ and $\tau_{\mathrm{est}}=5 \mathrm{~ms}$, the level of distortion is negligible. This is due to the fact that with increase in $P_{\text {rcvd }}, P_{\text {fa }}$ shifts to a very low value such that $1-\mathrm{P}_{\mathrm{fa}} \approx 1$. Moreover, for large $\gamma_{\mathrm{rcvd}}$, the sensing-throughput curvature shifts to the right leading to a low $\tilde{\tau}_{\text {sen }}^{\mathrm{P}}$, thereby making distortion in terms of $\tilde{\tau}_{\text {sen }}^{\mathrm{L}}, \tilde{\tau}_{\text {sen }}^{\mathrm{U}}$ insignificant. Hence, the system becomes more tolerant due to the reduced distortion in the system parameters. Besides that, by reducing $\tau_{\text {est }}$, 
i.e., $\tau_{\text {est }}<5 \mathrm{~ms}$, it is possible to minimize the margin between $\tilde{R}_{\mathrm{s}}$ and $\tilde{R}_{\mathrm{s}}^{\mathrm{P}}$, this however increases the level of distortion for IS. This way, for a given choice of $\tau_{\text {est }}$ and maximum distortion in the $\tilde{R}_{\mathrm{s}}^{\mathrm{P}}$, we can define an operation regime for IS in terms of $\gamma_{\mathrm{rcvd}}$, for example operation regime with $\tau_{\text {est }}=5 \mathrm{~ms}$ is defined as $\gamma_{\mathrm{rcvd}} \geq-5 \mathrm{~dB}$, cf. Fig. 3a. Hence, an extension of this regime below $-5 \mathrm{~dB}$ can be attained by increasing the estimation time beyond $5 \mathrm{~ms}$.

In addition to the $\tilde{R}_{\mathrm{s}}^{\mathrm{P}}$, it is also important to depict the distortion in $\mathrm{P}_{\mathrm{d}}$. The distortion in $\mathrm{P}_{\mathrm{d}}^{\mathrm{P}}$ is characterized using (16). Clearly, $\mathrm{P}_{\mathrm{d}}^{\mathrm{P}}$ remains constant, i.e., $\mathrm{P}_{\mathrm{d}}^{\mathrm{P}}=\overline{\mathrm{P}}_{\mathrm{d}}$ with $\tau_{\text {est }}$ and for $\gamma_{\mathrm{rcvd}} \in\{-10,-5,0\} \mathrm{dB}$. Fig. 3b reveals the distortion in $\mathrm{P}_{\mathrm{d}}^{\mathrm{P}}$ in terms of $\mathrm{P}_{\mathrm{d}}^{\mathrm{L}}$ and $\mathrm{P}_{\mathrm{d}}^{\mathrm{U}}$. It is evident that the distortion is small for $\mathrm{a}$ large value of $\gamma_{\mathrm{rcvd}}$ and it decreases with increase in $\tau_{\text {est }}$. Most importantly, it is observed that an $\mathrm{P}_{\mathrm{d}}^{\mathrm{U}}$ depicted from $P_{\mathrm{rcvd}}^{\mathrm{U}}$ forms a lower bound whereas $\mathrm{P}_{\mathrm{d}}^{\mathrm{L}}$ forms an upper bound to $\mathrm{P}_{\mathrm{d}}^{\mathrm{P}}$. It is clear from the fact that, the distortion in $\bar{P}_{\text {rcvd }} \leq P_{\text {rcvd }}^{\mathrm{U}}$ shifts the threshold to its right side $\epsilon \geq \epsilon^{\mathrm{U}}$, whereas the probability density function corresponds to hypothesis $\mathcal{H}_{1}$ has a fixed expression subject to $\bar{P}_{\text {rcvd }}$, hence, this shift in threshold causes the probability of detection to decrease, i.e., $\mathrm{P}_{\mathrm{d}}^{\mathrm{U}} \leq \mathrm{P}_{\mathrm{d}}^{\mathrm{P}}$. Similarly, $\epsilon^{\mathrm{L}} \leq \epsilon$ corresponds to upper bound $\mathrm{P}_{\mathrm{d}}^{\mathrm{P}} \leq \mathrm{P}_{\mathrm{d}}^{\mathrm{L}}$.

From the perspective of the secondary user, it is interesting to depict the distortion in $\mathrm{P}_{\mathrm{fa}}^{\mathrm{P}}$. Fig. 4a analyzes the distortion in $\mathrm{P}_{\mathrm{fa}}^{\mathrm{P}}$ according to (17) versus $\tau_{\text {est }}$ for $\gamma_{\mathrm{rcvd}}=\{-10,-5,0\} \mathrm{dB}$. Clearly, $\mathrm{P}_{\mathrm{fa}}^{\mathrm{P}}$ decreases with increase in $\gamma_{\mathrm{rcvd}}$ and remains constant with $\gamma_{\text {rcvd }}$. Analog to $\mathrm{P}_{\mathrm{d}}^{\mathrm{P}}, \mathrm{P}_{\text {fa }}^{\mathrm{U}} \leq \mathrm{P}_{\text {fa }}^{\mathrm{P}} \leq \mathrm{P}_{\text {fa }}^{\mathrm{L}}$, hence, $\mathrm{P}_{\text {fa }}^{\mathrm{L}}$ and $\mathrm{P}_{\text {fa }}^{\mathrm{U}}$ form an upper and lower bounds, respectively.

Apart from the performance parameters, the optimum sensing time $\tilde{\tau}_{\text {sen }}^{\mathrm{P}}$ is an important system parameter that is closely related to the performance parameters. Hence, Fig. 4b reveals the distortion in the $\tilde{\tau}_{\text {sen }}^{\mathrm{P}}$ versus the $\tau_{\text {est }}$ for $\gamma_{\mathrm{rcvd}} \in\{-10,-5,0\} \mathrm{dB}$, cf. (15). Similar to $\mathrm{P}_{\mathrm{d}}^{\mathrm{P}}$ and $\mathrm{P}_{\mathrm{fa}}^{\mathrm{P}}, \tilde{\tau}_{\text {sen }}^{\mathrm{L}}$ and $\tilde{\tau}_{\text {sen }}^{\mathrm{U}}$ represent the upper and lower bound to $\tilde{\tau}_{\text {sen }}^{\mathrm{P}}$. It is obvious from the fact that larger value of estimated received power $P_{\text {rcvd }}^{\mathrm{U}}$ shifts the curvature in the sensingthroughput, that depicts the optimum sensing time, to a lower value, therefore, $\tau_{\text {sen }}^{\mathrm{U}} \leq \tau_{\text {sen }}^{\mathrm{P}} \leq \tau_{\text {sen }}^{\mathrm{L}}$, cf. Fig. 4 b.

\section{Conclusion}

In this paper, we considered the deployment of a cognitive radio as an interweave system. For sustaining a minimum probability of detection, it requires the knowledge of the received power at the ST. To acquire this knowledge, an estimation has been included within the frame duration. In this regard, we proposed an estimation model that characterizes the actual performance of the IS. More specifically, the distortion in terms of bounds on the performance parameters has been captured based on the analytical expression. Moreover, it has been indicated that the severity in distortion can be confined by regulating the estimation time. Through theoretical and numerical analysis, it has been demonstrated that for a given choice of estimation time, the distortion in the performance parameters limits the operation regime, defined in terms of the received signal to noise ratio, at the $\mathrm{ST}$. 
In future, we plan to depict the exact expression of distortion instead of performance bounds. To pursue this, an outage constraint will be applied on the probability of detection in place of received power, that is $\mathrm{P}\left(\mathrm{P}_{\mathrm{d}} \leq \overline{\mathrm{P}}_{\mathrm{d}}\right)$. In this way, we shall determine the estimation time subject to the new outage constraint.

\section{References}

[1] Goldsmith, A., Jafar, S., Maric, I., Srinivasa, S.: Breaking Spectrum Gridlock With Cognitive Radios: An Information Theoretic Perspective. Proceedings of the IEEE 97(5) (May 2009) 894-914

[2] Axell, E., Leus, G., Larsson, E., Poor, H.: Spectrum sensing for cognitive radio : State-of-the-art and recent advances. IEEE Signal Processing Magazine 29(3) (May 2012) 101-116

[3] Sharma, S., Chatzinotas, S., Ottersten, B.: Exploiting polarization for spectrum sensing in cognitive satcoms. In: CROWNCOM. (June 2012) 36-41

[4] Urkowitz, H.: Energy detection of unknown deterministic signals. Proceedings of the IEEE 55(4) (april 1967) $523-531$

[5] Kostylev, V.: Energy detection of a signal with random amplitude. In: ICC. Volume 3. (2002) 1606-1610 vol.3

[6] Digham, F., Alouini, M.S., Simon, M.K.: On the energy detection of unknown signals over fading channels. In: ICC. Volume 5. (May 2003) 3575-3579 vol.5

[7] Herath, S., Rajatheva, N., Tellambura, C.: Unified approach for energy detection of unknown deterministic signal in cognitive radio over fading channels. In: ICC Workshops. (June 2009) 1-5

[8] Mariani, A., Giorgetti, A., Chiani, M.: Energy detector design for cognitive radio applications. In: 2010 International Waveform Diversity and Design Conference (WDD). (Aug 2010) 000053-000057

[9] Tandra, R., Sahai, A.: SNR Walls for Signal Detection. IEEE Journal of Selected Topics in Signal Processing 2(1) (Feb 2008) 4-17

[10] Liang, Y.C., Zeng, Y., Peh, E., Hoang, A.T.: Sensing-Throughput Tradeoff for Cognitive Radio Networks. IEEE Transactions on Wireless Communications 7(4) (April 2008) 1326-1337

[11] Cardenas-Juarez, M., Ghogho, M.: Spectrum Sensing and Throughput Tradeoff in Cognitive Radio under Outage Constraints over Nakagami Fading. IEEE Communications Letters 15(10) (October 2011) 1110-1113

[12] Sharkasi, Y., Ghogho, M., McLernon, D.: Sensing-throughput tradeoff for OFDMbased cognitive radio under outage constraints. In: ISWCS. (Aug 2012) 66-70

[13] Kaushik, A., Sharma, S.K., Chatzinotas, S., Ottersten, B., Jondral, F.K.: Estimation-Throughput tradeoff for underlay cognitive radio systems. In: IEEE Int. Conf. on Communications (ICC) - Cognitive Radio and Networks Symposium. (June 2015) (to appear).

[14] Kaushik, A., Mueller, M., Jondral, F.K.: Cognitive Relay: Detecting Spectrum Holes in a Dynamic Scenario. In: ISWCS. (Apr. 2013) 1-2

[15] Kay, S.: Fundamentals of Statistical Signal Processing: Detection theory. Prentice Hall Signal Processing Series. Prentice-Hall PTR (1998)

[16] Gradshteyn, I.S., Ryzhik, I.M.: Table of Integrals, Series, and Products. Sixth edn. San Diego, CA: Academic Press. (2000) 